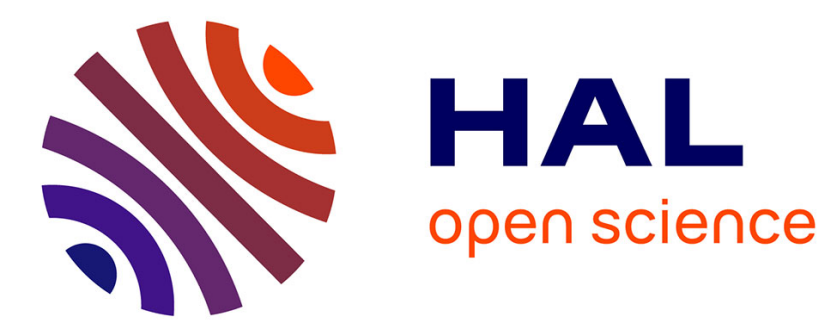

\title{
Style de leadership, LMX et engagement organisationnel des salariés : le genre du leader a-t-il un impact ?
}

Sarah Saint-Michel, Nouchka Wielhorski

\section{To cite this version:}

Sarah Saint-Michel, Nouchka Wielhorski. Style de leadership, LMX et engagement organisationnel des salariés : le genre du leader a-t-il un impact ?. @GRH, 2011, 1 (1), 10.3917/grh.111.0013 . hal-01907955

\section{HAL Id: hal-01907955 \\ https: / hal-paris1.archives-ouvertes.fr/hal-01907955}

Submitted on 29 Oct 2018

HAL is a multi-disciplinary open access archive for the deposit and dissemination of scientific research documents, whether they are published or not. The documents may come from teaching and research institutions in France or abroad, or from public or private research centers.
L'archive ouverte pluridisciplinaire HAL, est destinée au dépôt et à la diffusion de documents scientifiques de niveau recherche, publiés ou non, émanant des établissements d'enseignement et de recherche français ou étrangers, des laboratoires publics ou privés. 


\section{STYLE DE LEADERSHIP, LMX ET ENGAGEMENT ORGANISATIONNEL DES SALARIÉS : LE GENRE DU LEADER A-T-IL UN IMPACT ?}

Sarah Saint-Michel, Nouchka Wielhorski

De Boeck Supérieur | « @GRH »

2011/1 n²1 pages 13 à 38

ISSN 2034-9130

ISBN 9782804166991

Article disponible en ligne à l'adresse :

https://www.cairn.info/revue-@grh-2011-1-page-13.htm

\section{Pour citer cet article :}

Sarah Saint-Michel, Nouchka Wielhorski« Style de leadership, LMX et engagement organisationnel des salariés : le genre du leader a-t-il un impact ? », @GRH 2011/1 $\left(\mathrm{n}^{\circ} 1\right)$, p. 13-38.

DOI 10.3917/grh.111.0013

Distribution électronique Cairn.info pour De Boeck Supérieur.

(C) De Boeck Supérieur. Tous droits réservés pour tous pays.

La reproduction ou représentation de cet article, notamment par photocopie, n'est autorisée que dans les limites des conditions générales d'utilisation du site ou, le cas échéant, des conditions générales de la licence souscrite par votre établissement. Toute autre reproduction ou représentation, en tout ou partie, sous quelque forme et de quelque manière que ce soit, est interdite sauf accord préalable et écrit de l'éditeur, en dehors des cas prévus par la législation en vigueur en France. Il est précisé que son stockage dans une base de données est également interdit. 


\title{
STYLE DE LEADERSHIP, LMXET ENGAGEMENT ORGANISATIONNEL DES SALARIÉS : LE GENRE DU LEADER A-T-IL UN IMPACT?
}

\author{
Sarah Saint-Michel \\ Laboratoire Prism Cergors \\ Université Paris 1 Panthéon Sorbonne \\ 17, rue de la Sorbonne, 75005 Paris \\ sarah.saint-michel@univ-paris1.fr

\section{Nouchka Wielhorski} \\ Laboratoire Prism Cergors \\ Université Paris 1 Panthéon Sorbonne \\ 17, rue de la Sorbonne, 75005 Paris \\ nouchka.wielhorski@univ-paris1.fr
}

Résumé

Cette recherche examine l'incidence du genre sur le style de leadership transformationnel et transactionnel des managers ainsi que sur la qualité de la relation LMX et sur l'engagement organisationnel des subordonnés. Elle a été menée auprès de 111 salariés, hommes et femmes, évaluant à la fois le style de leadership de leur supérieur et leur perception de la relation Leader-Member Exchange (LMX). Les résultats mettent en Iumière l'absence d'impact significatif du genre du dirigeant sur la perception du style de leadership et sur la qualité de la relation LMX. Toutefois, le genre du leader conjugué, notamment, au comportement "management par exception " du leadership transactionnel, a une incidence différente selon le type d'engagement organisationnel déployé par les subordonnés. Ainsi, lorsque les managers femmes adoptent ce type de comportement, leurs subordonnés tendent à manifester un engagement affectif moindre vis-à-vis de leur organisation. Quand les managers hommes exercent ce type de leadership, leurs subordonnés s'orientent plutôt vers un engagement organisationnel calculé et normatif. Les résultats seront interprétés au regard de la théorie du rôle social (Eagly, 1987). Cette 
étude tente d'apporter une contribution aux liens entre le genre, le style de leadership, la relation LMX et l'implication des salariés dans le contexte organisationnel français.

\section{Mots clés}

genre, leadership, relation LMX, engagement organisationnel.

\section{Abstract}

This research examines the gender impact on the transformational-transactional leadership style as well as on the Leader Member Exchange relationship and its effects on the follower's organisational commitment. This study has been carried out with 111 followers, female and male assessing their manager's leadership style of their LMX perception. Results indicate that the leader's gender has no impact on the perception of the manager's leadership style neither on the LMX quality. However the leader's gender especially combined with the dimension "management by exception" of leadership transactional has a different impact according to the type of follower's organizational commitment. Thus, when female leaders adopt this type of leadership, the follower's organisational commitment tends to be negatively affective. When male leaders adopt this type of leadership, the follower's organisational commitment tends to be normative and continuous. Results will be interpreted according to the social role theory (Eagly, 1987). This research attempts to contribute to the study of relationship between gender, leadership style, LMX and the organizational commitment of followers in the French organizational context.

\section{Keywords}

gender, leadership, LMX, organizational commitment. 


\section{INTRODUCTION}

Au cours des trois dernières décennies, de nombreuses évolutions politiques ont contribué à lever le voile sur le travail des femmes, notamment en France, par le biais des lois sur l'égalité professionnelle ${ }^{1}$. Elles accèdent plus facilement aux formations longues : une étude américaine révèle qu'elles représentent près de la moitié des diplômés en comptabilité, en gestion des entreprises ou en droit (Catalyst, 1999, 2000). Les femmes qualifiées entrent sur le marché du travail à un niveau comparable à celui des hommes, présentent les mêmes attentes, mais les itinéraires professionnels divergent rapidement (Davidson et Burke, 2000) : elles accèdent en effet difficilement aux positions stratégiques des organisations. Tandis qu'elles constituent environ $50 \%$ de la population active, elles n'occupent que $33 \%$ des postes de management intermédiaire, et seulement $7 \%$ d'entre elles se positionnent sur des fonctions dirigeantes au sein des entreprises ou des administrations publiques (Blanchard et al., 2009 ; Observatoire de la Parité, 2010). Cependant, la mise au jour du phénomène du plafond de verre a opéré une réflexivité nécessaire à une évolution sociétale : les femmes accèdent aujourd'hui davantage aux postes à responsabilité. Toutefois, malgré ce changement positif, des disparités liées au genre persistent dans les entreprises.

Cette communication tentera d'apporter des éléments de compréhension relatifs à ce phénomène. Les femmes adoptent-elles un style de management différent de celui des hommes? Serait-ce la raison pour laquelle elles accèdent peu aux positions hiérarchiques supérieures? Les incidences du genre sur le style de leadership et sur l'engagement organisationnel des salariés seront appréhendées dans cette étude. En effet, de nombreuses recherches ont démontré l'impact positif de l'engagement organisationnel des salariés : baisse de l'intention de quitter l'entreprise, augmentation de la satisfaction au travail, diminution de l'absentéisme (Tett et Meyer, 1993). Plus précisément, le genre du leader a-t-il un impact différencié sur le type d'engagement organisationnel déployé par les salariés?

Si la recherche anglo-saxonne s'est intéressée aux liens entre genre et leadership, il n'existe, en France, aucune étude abordant cette thématique. Afin d'initier cette analyse dans le contexte organisationnel français, la complexité des liens entre genre et leadership sera évoquée dans un premier temps ; ensuite, l'engagement organisationnel des salariés sera approfondi ; enfin, les résultats seront présentés puis discutés.

[1] Loi Roudy 1983 et loi Génisson 2001 sur l'égalité professionnelle. 


\section{LE CADRE THÉORIQUE DU LEADERSHIP : FULL RANGE LEADERSHIP MODEL ET RELATION LMX}

\section{, Leadership transformationnel et transactionnel}

Depuis le début des années 1980, de nombreuses recherches ont mis l'accent sur les caractères personnels du leader. Elles s'inspirent des travaux de James Mc Gregor Burns (1978) qui, dans son ouvrage intitulé Leadership, distingue deux styles de leadership politique : le leadership transactionnel et le leadership transformationnel. Les approches transactionnelles s'intéressent aux échanges s'inscrivant dans une logique de transaction entre le manager et le subordonné. Un leader transactionnel s'attache « aux moyens de maintenir et d'améliorer le niveau ainsi que la qualité de la performance de ses subordonnés ". D'après Burns, cette conception tranche avec l'approche transformationnelle selon laquelle le leadership est un processus qui provoque un engagement mutuel du leader et du subordonné placé sous sa dépendance hiérarchique. Cet engagement repose sur l'adhésion commune à une vision et à des valeurs partagées avec le leader.

Les recherches menées par Burns ont ensuite été reprises par Bass (1985), qui les a étendues au contexte organisationnel. Ce dernier (1985) s'est particulièrement intéressé aux comportements manifestés par les leaders. II distingue également deux styles de leadership : le leadership transformationne/ et le leadership transactionnel. Mais, selon lui, un même leader peut se comporter à la fois de manière transactionnelle et transformationnelle. II conteste donc l'opposition de Burns entre ces deux types de leadership. Loin de représenter les extrémités d'un même continuum, le leadership transactionnel et transformationnel sont des concepts différents. Bass (1985) affirme même qu'un bon manager est à la fois transactionnel et transformationnel. Un leader transformationnel est perçu comme le prolongement d'un leader transactionnel. Ainsi, le dirigeant transformationnel " consacre son énergie à faire prendre conscience à ses collaborateurs de l'importance des conséquences des actions engagées ". Cela nécessite qu'il fasse preuve de charisme et de confiance en soi afin de faire partager sa vision à son équipe, au risque d'être parfois en contradiction avec la pensée dominante du moment (Bass, 1985 ; Neveu, 2004). La capacité à obtenir l'adhésion volontaire des subordonnés, sans promesse de récompense, constitue un trait spécifique du leader transformationnel. Quel que soit le niveau hiérarchique, le leadership transformationnel peut être observé (Bass, 1985). Le leader transformationnel s'incarne comme étant un modèle à suivre de la part de ses collaborateurs et gagne ainsi leur confiance. Par ses actions de délégation de pouvoir et de mentoring, le dirigeant obtient de ses subordonnés qu'ils transcendent leur propre potentiel, contribuant ainsi à la performance organisationnelle. Tandis que le leader transactionnel centre sa relation de travail avec son subordonné sur un échange de ressources, le leader transformationnel fournit aux employés ce qu'ils souhaitent (i.e. 
des récompenses contingentes) en échange d'objectifs imposés par le leader (Kuhnert et Lewis, 1987). Ce type de leadership implique un style de management plus instrumental, où la nature des relations entre le leader et son équipe est de type donnant-donnant.

Nous venons d'exposer ce que les chercheurs nomment la théorie du Full Range Leadership Model (Bass et Avolio, 1991). Dans la section suivante, nous aborderons les théories plus récentes du leadership, en nous concentrant davantage sur la relation nouée entre le leader et ses subordonnés (Neveu, 2004 ; Northouse, 2010).

\section{, La relation leader-member exchange (LMX)}

Deux perspectives sur le leadership prévalent dans la littérature académique gestionnaire. La première est centrée sur les traits et les comportements du leader, éléments qui expliquent la performance de l'individu ou du groupe de travail, en reliant directement le comportement du leader à la performance. Ce point de vue a été examiné précédemment avec le Full Range Leadership Model (Bass et Avolio, 1991). La seconde se focalise sur la création d'une relation d'échange entre le manager et son subordonné formant une véritable dyade. Cette approche, mise en avant par Graen et Uhl-bien (1995), se nomme la théorie LMX (Leader-Member-Exchange). Une définition a été proposée par Scandura et Graen (1986) : le LMX désigne " un système de "constituants" ainsi que les relations entretenues entre les deux membres d'une dyade générant certains types de comportements interdépendants et partageant des résultats mutuels, favorisant une certaine conception de l'environnement, des valeurs et des cartes de "causalité" ". Ainsi, le leader communique à ses subalternes une attente de rôle (role expectation) puis leur fournit des récompenses tangibles et intangibles, qui satisfont les attentes réalisées ou pas (Hui et al., 2004). Émerge alors un processus réciproque dans l'échange dyadique, entre le leader et ses collaborateurs, où chaque partie apporte des ressources différentes. Les rôles attendus du leader et du subordonné sont négociés en permanence. C'est cette négociation qui définit la qualité et la maturité de la relation LMX. L'échange interpersonnel entre le leader et ses salariés va déterminer le type de rôle que le subordonné jouera au sein de l'unité de travail.

Dans cette perspective, la relation entre un salarié et son supérieur est susceptible de donner lieu à des échanges de " faible qualité » caractérisés par le recours à l'autorité organisationnelle formelle. Le leader obtient de son subordonné une « performance de routine ", lequel retire, en échange, de la relation des " bénéfices organisationnels standards » (Graen et Cashman, 1975 ; Neveu, 2004). Dans ce prolongement, la relation LMX peut s'avérer de " bonne qualité ", transcendant ainsi les objectifs initialement fixés entre le leader et son subordonné. Graen et Uhl-Bien (1991) indiquent que la relation LMX se développe au travers de trois périodes. Une phase « inconnue " au cours de laquelle le leader offre la possibilité d'élargir les rôles ainsi que les responsabilités du salarié et évalue ses compétences (1). Pendant la phase de connaissance, leader et subordonné 
apprennent à se comprendre et à développer une confiance mutuelle (2). Enfin, pendant la phase d'« associé » la relation LMX, est dite " forte " dans la mesure où le lien hiérarchique a disparu (3). II s'agit alors d'une relation LMX aboutie. Graen et Uhl-Bien (1995) signalent que chacune de ces phases est davantage reliée au leadership transformationnel qu'au leadership transactionnel. Le leader transformationnel, grâce à son charisme, permet aux salariés de s'identifier à lui ; ceux-ci acceptent, par conséquent, plus facilement un élargissement de leurs responsabilités dans l'entreprise. La relation LMX s'intensifie ; la motivation des salariés augmente, entraînant ainsi un dépassement de leurs propres objectifs au profit de ceux de l'organisation.

En définitive, il existe une forte corrélation entre le leadership transformationnel et la relation LMX; à l'inverse, la relation LMX perd en intensité lorsque le leader déploie un leadership de type transactionnel.

\section{FULL RANGE LEADERSHIP MODEL ET RELATION LMX : LE GENRE DU LEADER A-T-IL UN IMPACT?}

Les études portant sur les liens entre genre et style de leadership ont conduit à des résultats contradictoires. Les partisans du " oui » et les partisans du " non " s'opposent (Cornet et Bonnivert, 2008). Nous présenterons successivement les différents courants avant de mettre en perspective nos hypothèses de recherche.

\section{, Le genre du leader n'a aucun impact sur le style de leadership déployé}

L'idée selon laquelle le genre a un impact sur le style de leadership n'est pas unanimement partagée ; des recherches viennent nuancer l'importance de cette variable. Ainsi, Maccoby et Jacklin (1974) notent que les études centrées sur la thématique du lien entre genre et leadership ne relèvent aucune corrélation entre les deux notions. Ces propos sont vérifiés empiriquement ; Eagly et Johnson (1990) soulignent que les facteurs organisationnels modèrent l'émergence d'éventuelles différences associées au genre sur le style de leadership. Celles-ci seraient liées aux caractéristiques du contexte organisationnel dans lequel le leadership est déployé. De manière similaire, Maher (1997) ne reporte aucune différence liée au genre lors de l'évaluation d'hommes et de femmes leaders sur l'utilisation du leadership transformationnel et transactionnel. De même, Kolb (1999) souligne que deux décennies de recherche sur cette thématique n'ont pas permis d'observer de différences comportementales significatives entre les hommes et les femmes leaders. Ces assertions ont été validées empiriquement par van Engen et al. (2001) : le sexe du manager n'a aucun impact sur le style de leadership transformationnel, et ce, quel que soit le contexte organisationnel. 
Ces chercheurs, qui s'appuient sur la théorie structurelle (Kanter, 1977) précisent que le rôle de leader implique un certain type de leadership, intériorisé aussi bien chez les hommes que chez les femmes exerçant les mêmes rôles et se comportant de manière similaire (Acker, 1990 ; Kanter, 1977 ; Komives, 1991 ; Mahar, 1997 ; Nieva et Gutek, 1981 ; van Engen et al., 2001 ; Vecchio, 2002 ; Wajcman, 1998). Cet argument naît du principe selon lequel les hommes et les femmes ont dû affronter les mêmes difficultés pour accéder aux postes de direction. Par conséquent, un leader qui occupe une telle position hiérarchique doit remplir les attentes associées à son rôle de pouvoir. Ceci modélise et oriente son action dans une direction particulière (Phillips et Lord, 1981). Cette pression des comportements similaires entre hommes et femmes leaders conduit à l'absence de dissemblance liée au genre sur le style de leadership. Néanmoins, si des disparités existent, elles s'avèrent relativement faibles. Ainsi, les femmes et les hommes leaders ont assimilé et intériorisé les codes sociaux afin de se conformer au rôle de leader ; ce qui explique cette similitude des conduites. Les rôles de dirigeant sont, le plus souvent, clairement définis en termes d'attentes comportementales. Ces managers ont été socialisés pour atteindre ces attentes dès qu'ils ont été identifiés comme susceptibles d'exercer un jour une fonction de responsabilité (Eagly et Johnson, 1990 ; Feldman, 1976 ; Graen, 1976 ; Terborg, 1977 ; Wanous, 1977).

Cependant, d'autres recherches mettent en évidence des différences liées au genre sur le style de leadership.

\section{, Le genre du leader a un impact sur le style de leadership transformationnel}

L'argument principal, soutenant que le genre influe sur le style de leadership, se fonde sur le processus de socialisation. Les tenants de ce courant allèguent que, durant ce processus, les femmes ont développé des caractéristiques différentes des valeurs dites " traditionnelles " de leadership telles que la compétition, le contrôle et les comportements agressifs (Helgesin, 1990 ; Pounder et al., 2002 ; Rosener, 1990). Eagly (1987) souligne que les individus se comportent conformément aux attentes sociales prescrites par leur rôle. Nous intériorisons donc les codes culturels de notre rôle social en adéquation avec notre sexe et, par conséquent, sommes intrinsèquement motivés à agir en fonction de celui-ci (Eagly, 1987 ; Eagly et al., 2003). Ainsi, il est attendu des femmes qu'elles soient tournées vers les relations interpersonnelles, vers des missions de soutien, tandis que les hommes devraient se comporter en dominateurs, centrés sur la tâche. Afin d'étayer ces arguments théoriques, des recherches empiriques ont été menées. Ainsi Kouzes et Posner (1990), s'appuyant sur leur modèle de leadership transformationnel ${ }^{2}$, révèlent que les femmes leaders sont plus aptes que les hommes à déployer des

[2] Leur instrument de mesure pour évaluer le leadership transformationnel est le Leadership Practice Inventory (LPI). 
comportements de type transformationnel ${ }^{3}$. De même, Rosener (1990), via une autoévaluation de managers hommes et femmes, démontre que celles-ci tendent à se décrire comme davantage proches de comportements transformationnels que leurs homologues masculins. Druskat (1994), au travers d'une étude réalisée dans le milieu des Églises catholiques, avance des résultats semblables. Ces conclusions ont été confirmées, par la suite, dans un contexte managérial, sur un échantillon composé uniquement de femmes. Ainsi Yammarino et al. (1997) relèvent qu'elles tendent à développer une relation unique avec leurs subordonnés, notamment par le biais de considérations individualisées. De même Carless (1998), à l'aide d'une évaluation à 360 degrés, précise que les supérieurs et les femmes leaders s'auto-évaluent de manière plus transformationnelle que leurs homologues masculins. Cependant, les subordonnés apprécient de manière identique le style de leadership de leur supérieur hiérarchique.

Des recherches plus récentes signalent également des divergences liées au genre sur le style de leadership. Ainsi, Mandell et Pherwani (2003) dévoilent que les femmes leaders présentent des scores plus élevés que les hommes sur le leadership transformationnel. Ces résultats ont été confirmés grâce à une méta-analyse réalisée par Eagly et al. en 2003. Celle-ci repose sur 45 études ayant évalué le style de leadership transformationnel, transactionnel et le laissez-faire, des deux sexes, en position de leadership, soit par auto-évaluation du leader (36 \% des études recensées), soit à l'aide d'une évaluation par les subordonnés, pairs ou supérieurs hiérarchiques. Les résultats montrent que les femmes leaders tendent un peu plus que les hommes à déployer un style de leadership transformationnel. Ainsi, les femmes en position de leadership adoptent à $52,5 \%$ des comportements de leadership transformationnel contre $47,5 \%$ des hommes managers. Les femmes pratiquent davantage un style de leadership transformationnel, notamment sur les sous-dimensions " charisme ", " stimulations intellectuelles » et " considérations individualisées " ainsi qu'un leadership transactionnel, particulièrement sur la sous-dimension " contingence des récompenses". Les hommes leaders obtiennent des scores plus élevés que les femmes sur les sous-dimensions du leadership transactionnel, " laissez-faire " et "management par exception actif et passif ". II est important de noter que, la taille de l'effet $(d=-.11)^{4}$, laquelle souligne la différence liée au genre sur le style de leadership transformationnel, reste faible (Vecchio, 2002). Cette différence de 5 points suggère une légère tendance des femmes à fournir un style de leadership plus transformationnel que les hommes.

[3] Les comportements transformationnels renvoient aux sous-dimensions suivantes : " modelling the way " (i.e. fournir une vision claire et convaincante du futur) et " encouraging the heart " (i.e. fournir un retour d'information sur les actions menées par les subordonnés).

[4] Une taille d'effet positive ( $d$ ) indique que les hommes leaders présentent un score plus élevé que les femmes sur le style de leadership ; une taille d'effet négative $(d)$ indique que les femmes leaders ont un score plus élevé que les hommes sur le style de leadership (Eagly et al., 2003 ; Hunter et Schmidt, 1980). 
En nous appuyant sur la revue de littérature ainsi que sur la méta-analyse réalisée par Eagly et al. (2003), nous posons les hypothèses suivantes :

Hypothèse 1 : Les sous-dimensions " charisme ", "stimulations intellectuelles " et " considérations individualisées" du leadership transformationnel sont positivement corrélées au leadership déployé par les femmes leaders.

Hypothèse 2 : La sous-dimension "contingence des récompenses " du leadership transactionnel est positivement corrélée au leadership déployé par les femmes leaders.

Hypothèse 3 : La sous-dimension "management par exception" du leadership transactionnel est corrélée positivement au leadership mis en œuvre par les hommes leaders.

\section{, La relation LMX au regard du genre du leader}

Si le genre est une variable susceptible de modérer le style de leadership, en est-il de même avec la relation LMX ? Le genre a-t-il un impact sur la qualité de la relation LMX ? Ceci n'a pas été clairement identifié comme étant corrélé positivement au genre du leader (Gerstner et Day, 1997). Cependant, se fondant sur les nombreux arguments théoriques des divergences liées au genre sur le style de leadership, certains chercheurs avancent que les femmes seraient plus enclines à favoriser et entretenir des relations de haute qualité avec leurs subordonnés (Vecchio, 2007).

En nous appuyant sur la théorie du rôle social (Eagly, 1987), selon laquelle les individus auraient tendance à agir en adéquation avec leur rôle de genre, culturellement défini, nous avançons que les femmes leaders auraient davantage de dispositions à développer une relation LMX de haute qualité avec leurs collaborateurs. D'après cette théorie, les femmes seraient stéréotypées comme étant " communautaires " et les hommes « agentiques " (Diekman et Eagly, 2000 ; Eagly, 1987). Les caractéristiques communautaires évoquent des attributs tels que la compassion, la générosité, le sentimentalisme, la tendresse ; alors que les caractéristiques agentiques décrivent des propriétés telles que la domination, l'agressivité, l'ambition, l'indépendance et la confiance en soi (Saint-Michel, 2010). Les stéréotypes de genre se manifestent particulièrement lors de l'attribution des habilités au leadership qui vont influer sur les attentes des subordonnés vis-à-vis de leur leader. Ainsi, nous postulons que les femmes leaders seraient plus disposées à déployer une relation LMX de haute qualité avec leurs subordonnés car des pressions sociales, externes aux individus, accentueraient le rôle de genre. Ainsi, ces pressions favoriseraient des comportements conformes à un rôle de genre. Comme le notent Eagly et al. (2003), ceci s'avère, dans une certaine mesure, problématique pour les femmes positionnées dans des fonctions de pouvoir car l'adéquation de leur rôle de genre féminin avec les stéréotypes masculins n'est pas appropriée. Les chercheurs nomment ce phénomène " l'incongruité du rôle de genre » (Eagly, 1987 ; Heilman, Block, Martell et Simon, 1988 ; Schein, 1973). Afin de résoudre ce problème de conflit de rôles, les femmes en position 
de leadership auraient plus tendance à adopter des comportements transformationnels, et à accroître les relations d'échange, ce qui leur permettrait de surmonter le dilemme de I'incongruité du rôle de genre (Eagly, 2003). Ainsi, une relation LMX de haute qualité avec leurs collaborateurs, fournirait aux femmes le moyen d'éviter les écueils trop masculins, en diminuant le dilemme de l'incongruité du rôle ; phénomène exacerbé lorsque celles-ci adoptent des comportements de commandement, d'autorité, perçus comme masculins (Eagly et al., 2003 ; Eagly et Karau, 2002 ; Yoder, 2001). Dans la lignée de ces recherches, nous postulons que les femmes leaders seraient plus susceptibles de déployer une relation LMX de haute qualité avec leurs collaborateurs afin de pallier ce dilemme lié aux rôles de genre et de leader. Ces différents arguments théoriques nous amènent à postuler l'hypothèse suivante :

Hypothèse 4 : Les femmes leaders entretiennent une relation LMX de meilleure qualité avec leurs subordonnés que les hommes leaders.

Nous venons d'exposer les liens éventuels entre le genre, le style de leadership et la relation LMX. Nous tenterons de cerner, dans la partie suivante, I'impact potentiel sur l'engagement organisationnel des salariés.

\section{LES LIENS ENTRE LE STYLE DE LEADERSHIP, LA RELATION LMX ET L'ENGAGEMENT ORGANISATIONNEL DES SUBORDONNÉS : LE GENRE DU LEADER A-T-IL UN IMPACT ?}

Nombre de chercheurs soulignent que les femmes détiendraient un " avantage au leadership " (Eagly et al., 2007). L'un des objectifs de cette communication est de comprendre si le genre du leader a effectivement un impact sur le type d'engagement organisationnel déployé par les subordonnés. L'engagement est considéré comme un output organisationnel important. II s'agit d'un objectif vers lequel les entreprises doivent tendre afin de fidéliser une main-d'œuvre motivée. L'engagement organisationnel est défini comme étant " une force générale conduisant l'individu à s'identifier et à s'engager envers l'organisation dans laquelle il travaille $»^{5}$ (Mowday et al., 1982, p. 27). Dans le cadre de cette communication, nous avons fait le choix de nous appuyer sur le concept d'engagement, modélisé par Allen et Meyer (1990). Ils proposent de distinguer trois dimensions de l'engagement organisationnel. Tout d'abord, I'engagement affectif se traduit par un attachement émotionnel et par l'identification à l'organisation. Ensuite,

[5] Expression traduite de la définition anglaise de Mowday et al. (1989) " the relative strength of an individual's identification with and involvement in a particular organization » (p. 27). 
l'engagement normatif repose sur une attitude de loyauté envers l'entreprise et/ou le supérieur hiérarchique ; celle-ci est dérivée d'un sentiment d'obligation morale à son égard. Enfin, l'engagement calculé s'appuie sur la perception du salarié quant aux coûts associés à une éventuelle rupture du lien contractuel avec son supérieur, voire son organisation. II fait référence à un calcul coût-risque associé au fait de rester ou pas dans l'organisation.

L'un des facteurs organisationnels, considéré comme déterminant de l'implication des subordonnés, est le style de leadership du supérieur (Mathieu et Zajac, 1990 ; Mowday et al., 1982). II existe un nombre important de recherches empiriques qui établissent une association positive entre le leadership transformationnel et l'engagement organisationnel dans différents contextes (Bono et Judge, 2003 ; Dumdum et al., 2002). Les travaux de Shamir et al. (1998) révèlent que les leaders transformationnels sont capables d'influencer le type d'engagement organisationnel de leurs subordonnés en diffusant un niveau élevé de valeurs intrinsèques, associées à l'atteinte d'objectifs. En effet, de nombreuses études révèlent des liens significatifs entre le leadership transformationnel et I'engagement affectif et normatif des salariés. Ainsi, Kent et Chelladurai (2001) affirment que les " considérations individualisées " sont corrélées positivement à l'engagement affectif et normatif. De même, Arnold et al. (2001) démontrent que les " stimulations intellectuelles » sont corrélées positivement à l'engagement affectif et normatif des salariés. De plus, la dimension « management par exception » du leadership transactionnel est corrélée positivement à l'engagement calculé des salariés (Hayward et al., 2004). Dans le prolongement de ces différentes études, nous proposons les hypothèses de recherche suivantes :

Hypothèse $\mathbf{5} \mathbf{a}$ : Le genre du leader modère la relation entre le style de leadership et l'engagement affectif des salariés. La relation est plus forte lorsque les leaders sont des femmes (en déployant des comportements transformationnels et de contingence des récompenses en vue de renforcer l'engagement affectif).

Hypothèse $\mathbf{5} \mathbf{b}$ : Le genre du leader modère la relation entre le style de leadership et l'engagement normatif des salariés. La relation est plus forte lorsque les leaders sont des femmes (en déployant des comportements transformationnels et de contingence des récompenses en vue de renforcer l'engagement normatif).

Hypothèse 6 : Le genre du leader modère la relation entre le style de leadership et l'engagement calculé des salariés. La relation est plus forte lorsque les leaders sont des hommes (en déployant des comportements de management par exception en vue de renforcer l'engagement calculé). 


\section{MÉTHODOLOGIE}

\section{, Participants et procédure}

Les données ont été collectées, en 2008, auprès de salariés issus de deux grandes entreprises : I'une spécialisée dans le secteur de la grande distribution d'articles de sport, l'autre dans la vente de produits cosmétiques (siège social). Ces données concernent des salariés dépendant d'un supérieur hiérarchique direct. Elles s'appuient sur les perceptions des sujets quant à leur relation avec leur $n+1$. Ainsi cette étude se concentre sur les représentations des subordonnés, quant au style de leadership de leur manager, la qualité de leur relation avec lui ainsi que leur implication vis-à-vis de l'organisation. Pour ce faire, le questionnaire a été administré, via un support papier, auprès de salariés volontaires. De manière à assurer la confidentialité des données, il était mentionné que celles-ci resteraient anonymes. Au total, près de 300 questionnaires ont été distribués auprès des deux entreprises; nous avons obtenu 111 questionnaires exploitables, soit un taux de retour d'environ $37 \%$. L'échantillon est composé de 60 répondants dépendant d'un manager de sexe masculin et de 51 répondants subordonnés à un manager de sexe féminin. L'ancienneté de la relation leader-salarié est d'environ 3 ans et demi.

\section{, Mesures}

\section{Le leadership transformationnel}

Le style de leadership transformationnel a été mesuré en utilisant l'échelle du Mutifactor Leadership Questionnaire Short Form 5X (ML0-5X) qui contient 18 items (Avolio et al., 1999 ; Bass et Avolio, 1997). Le leadership transformationnel inclut trois sous- échelles, à savoir : " le charisme ", "les stimulations intellectuelles " et " les considérations individualisées ». Un exemple d'item : «mon supérieur est un modèle à suivre pour moi ». Chaque item était mesuré à l'aide d'une échelle de Likert à 5 points allant de 1 (Pas du tout d'accord) à 5 (Tout à fait d'accord). Les différents coefficients alphas sont reportés au tableau 1. Pour la sous-dimension " charisme " $(\alpha=0,926)$; pour la sous-dimension "stimulation intellectuelle " $(\alpha=0,867)$ et pour la sous-dimension " considération individualisée " $(\alpha=0,759)$.

\section{Le leadership transactionnel}

Le style de leadership transactionnel a été mesuré en utilisant l'échelle du Multifactor Leadership Questionnaire Short Form 5X qui contient 8 items (Avolio et al., 1999 ; Bass et Avolio, 1997). Le leadership transactionnel comporte deux sous-échelles; à savoir : " la contingence des récompenses » (3 items) et le " management par exception » (5 items). Un exemple d'item: "Mon supérieur évoque régulièrement les récompenses (primes, promotion, etc.) potentielles que l'on peut obtenir en contrepartie d'un travail bien fait ». 
Chaque item était mesuré à l'aide d'une échelle de Likert à 5 points allant de 1 (Pas du tout d'accord) à 5 (Tout à fait d'accord). Les différents coefficients alphas sont reportés au tableau 2. Pour la sous-dimension " contingence des récompenses " $(\alpha=0,735)$ et pour la sous-dimension « management par exception » $(\alpha=0,725)$.

\section{La relation $L M X$}

S'agissant de l'évaluation de la qualité de la relation LMX entre le supérieur et le subordonné, nous avons fait le choix d'une approche unidimensionnelle du construit (Scandura et Graen, 1984). Les auteurs ont développé une échelle de mesure comportant 6 items. Son utilisation a été accompagnée de quelques préconisations. Scandura et Schriesheim (1994) recommandent notamment de faire remplir le questionnaire uniquement par le subordonné, et non par la dyade, comme cela a parfois été proposé (Graen et Uhl-Bien, 1995). Les qualités de fiabilité interne de l'échelle sont très satisfaisantes, I'alpha étant supérieur à 0,70 . Un exemple d'item : " Je sais généralement où j'en suis avec mon supérieur ". Chaque item était mesuré à l'aide d'une échelle de Likert à 5 points allant de 1 (Pas du tout d'accord) à 5 (Tout à fait d'accord). $(\alpha=0,901)$.

\section{L'engagement organisationnel}

L'engagement organisationnel a été mesuré via l'échelle proposée par Meyer et al. (1993). Celle-ci est composée de 18 items répartis en 6 items pour l'engagement affectif ; 6 pour l'engagement normatif et 6 pour l'engagement calculé. Un exemple d'item est « J'éprouve un vrai sentiment d'appartenance à mon entreprise ". Chaque item a été mesuré avec une échelle de Likert à 5 points allant de 1 (Pas du tout d'accord) à 5 (Tout à fait d'accord). Les différents alphas sont reportés au sein du tableau 3.

\section{Les variables de contrôle}

Durant l'étude, nous avons inclus les variables de contrôle suivantes : l'âge du leader, l'âge du subordonné, l'ancienneté dans l'entreprise du leader et l'ancienneté de la dyade formée entre le leader et le subordonné. Ces variables de contrôle ont été introduites dans le modèle de régression.

\section{, Analyse des données}

L'analyse des données a été réalisée en trois phases. Dans un premier temps, nous avons élaboré une analyse factorielle exploratoire, à l'aide du logiciel SPSS, afin de vérifier la structure factorielle des 4 variables étudiées. Nous avons également calculé le coefficient alpha de Cronbach afin d'évaluer la consistance interne des différentes échelles de mesure. Ensuite, nous avons réalisé des tests de comparaison de moyennes ( $t$-tests) pour déterminer les éventuelles différences, liées au genre, sur le style de leadership et pour tester nos hypothèses de recherche 1, 2, 3 et 4. Enfin, nous avons vérifié les hypothèses 5 et 6 par l'entremise des régressions linéaires hiérarchiques. 


\section{, Résultats}

Différence de moyennes entre les hommes et les femmes sur le style de leadership et le LMX

Afin de tester les hypothèses de recherche 1, 2, 3 et 4, nous avons réalisé un t-test ainsi qu'un test de différence de moyennes sur les multiples dimensions du leadership transformationnel et transactionnel, ainsi que sur la relation LMX (tableau 1). Les résultats du t-test et du test de différence de moyennes n'indiquent aucune différence significative liée au genre sur le style de leadership transformationnel, transactionnel et sur le LMX. Ainsi, les hypothèses de recherche 1,2, 3 et 4 sont rejetées ; ce qui signifie que le genre du leader n'a pas d'impact significatif sur le style de leadership ni sur la relation LMX. En dépit de légers écarts entre les moyennes obtenues par les femmes et les hommes sur les dimensions du leadership, aucune différence significative liée au genre ne peut être avancée. Les hypothèses 1, 2, 3 et 4 sont donc rejetées.

Tableau 1. Comparaison de l'évaluation des femmes et des hommes leaders par leurs subordonnés sur les dimensions du leadership transformationnel, transactionnel et sur la relation LMX

\begin{tabular}{|l|c|c|c|c|c|c|c|}
\hline \multicolumn{1}{|c|}{ Variables } & \multicolumn{2}{|c|}{$\begin{array}{c}\text { Femmes leaders } \\
(\boldsymbol{n}=\mathbf{5 1})\end{array}$} & \multicolumn{2}{|c|}{$\begin{array}{c}\text { Hommes leaders } \\
(\boldsymbol{n}=\mathbf{6 0})\end{array}$} & $\boldsymbol{t}$-test & $\boldsymbol{p}$ & $\begin{array}{c}\text { Différence } \\
\boldsymbol{d e} \\
\text { moyennes }\end{array}$ \\
\hline $\begin{array}{l}\text { Leadership } \\
\text { transformationnel }\end{array}$ & $\boldsymbol{M}$ & Ecart-type & $\boldsymbol{M}$ & Ecart-type & & & \\
\hline Charisme & 3,126 & 0,8886 & 3,289 & 0,8272 & 0,997 & n.s & 0,1625 \\
\hline $\begin{array}{l}\text { Stimulations } \\
\text { intellectuelles }\end{array}$ & 2,93 & 0,781 & 3,01 & 1,040 & 0,469 & n.s & 0,083 \\
\hline $\begin{array}{l}\text { Considérations } \\
\text { individualisées }\end{array}$ & 3,475 & 0,7941 & 3,321 & 0,8513 & $-0,984$ & n.s & $-0,1547$ \\
\hline $\begin{array}{c}\text { Leadership } \\
\text { transactionnel }\end{array}$ & & & & & & & \\
\hline $\begin{array}{l}\text { Contingence } \\
\text { des récompenses }\end{array}$ & 2,850 & 0,9026 & 2,822 & 1,0112 & $-0,150$ & n.s & $-0,0275$ \\
\hline $\begin{array}{l}\text { Management } \\
\text { par exception }\end{array}$ & 2,96 & 0,876 & 3,062 & 0,831 & 0,626 & n.s & 0,101 \\
\hline LMX & 3,363 & 0,918 & 3,614 & 0,912 & 1,441 & n.s & 0,2511 \\
\hline
\end{tabular}

Note : a $X$ homme $-X$ femme : un résultat positif indique que les hommes leaders ont un score moyen supérieur aux femmes leaders. 


\section{Corrélations entre le style de leadership, le LMX et l'engagement organisationnel des salariés}

Le tableau 2 présente les moyennes, les écarts-types et les corrélations entre le genre du leader, le style de leadership et l'engagement organisationnel des salariés. Les moyennes pour l'échelle du MLO sont similaires aux données reportées par Bass et Avolio (1997). Les résultats de ce tableau indiquent que le genre du supérieur hiérarchique est corrélé négativement et de manière significative à l'engagement organisationnel affectif $(r=-0,233 ; p<.05)$. Cette association négative entre le genre du supérieur hiérarchique et l'engagement affectif mérite d'être étudiée plus en profondeur. Les résultats signalent également que le charisme du leader est corrélé de manière significative avec la qualité de la relation LMX $(r=0,709 ; p<.01)$. Ainsi, plus les leaders sont perçus comme charismatiques, plus la relation LMX, entretenue avec leurs subordonnés, est de forte intensité. Concernant les liens entre le style de management et l'engagement organisationnel, notons que toutes les dimensions du leadership transformationnel sont corrélées de manière significative aux sous-dimensions de l'engagement. Le charisme du leader est corrélé de manière significative aux dimensions affective et calculée de l'engagement organisationnel $(r=0,564 ; p<.01$ et $r=0,216 ; p<.05)$. De même, la sous-dimension " stimulations intellectuelles " est corrélée de manière significative à l'engagement normatif $(r=0,449 ; p<.01)$. Enfin, la dimension " considération individualisée " est corrélée positivement à l'engagement calculé $(r=0,390 ; p<.01)$. Parmi les dimensions du leadership transactionnel, seule la dimension " contingence des récompenses " est corrélée significativement à l'engagement calculé des salariés ( $r=$ $0,408 ; p<.01)$. De même, le management par exception n'est pas significativement corrélé aux différentes dimensions de l'engagement organisationnel. Enfin, le construit LMX est corrélé significativement à l'engagement affectif et normatif des salariés ( $r=$ $0,417 ; p<.01 ; r=0,303 ; p<.01)$. Notons également que le LMX est corrélé de manière négative à la dimension " considération individualisée » $(r=-0,1 ; p>0,05)$. Ce résultat semble surprenant si l'on considère que plus le leader répond aux besoins individuels de ses collaborateurs, moins leur relation LMX est de bonne qualité. Ce résultat doit cependant être interprété avec précaution, puisqu'il n'est pas statistiquement significatif.

De manière à approfondir l'étude de nos résultats, nous avons procédé à une analyse par le biais de régressions linéaires multiples en introduisant les différentes variables explicatives.

\section{Résultats de la régression hiérarchique modérée : les déterminants de l'engagement organisationnel affectif, normatif et calculé}

Afin de mieux comprendre les déterminants de l'engagement organisationnel des salariés en fonction de la variable " genre " du leader, une régression hiérarchique modérée a été réalisée (Aguinis et Gottfredson, 2010). Suivant les recommandations émises par 


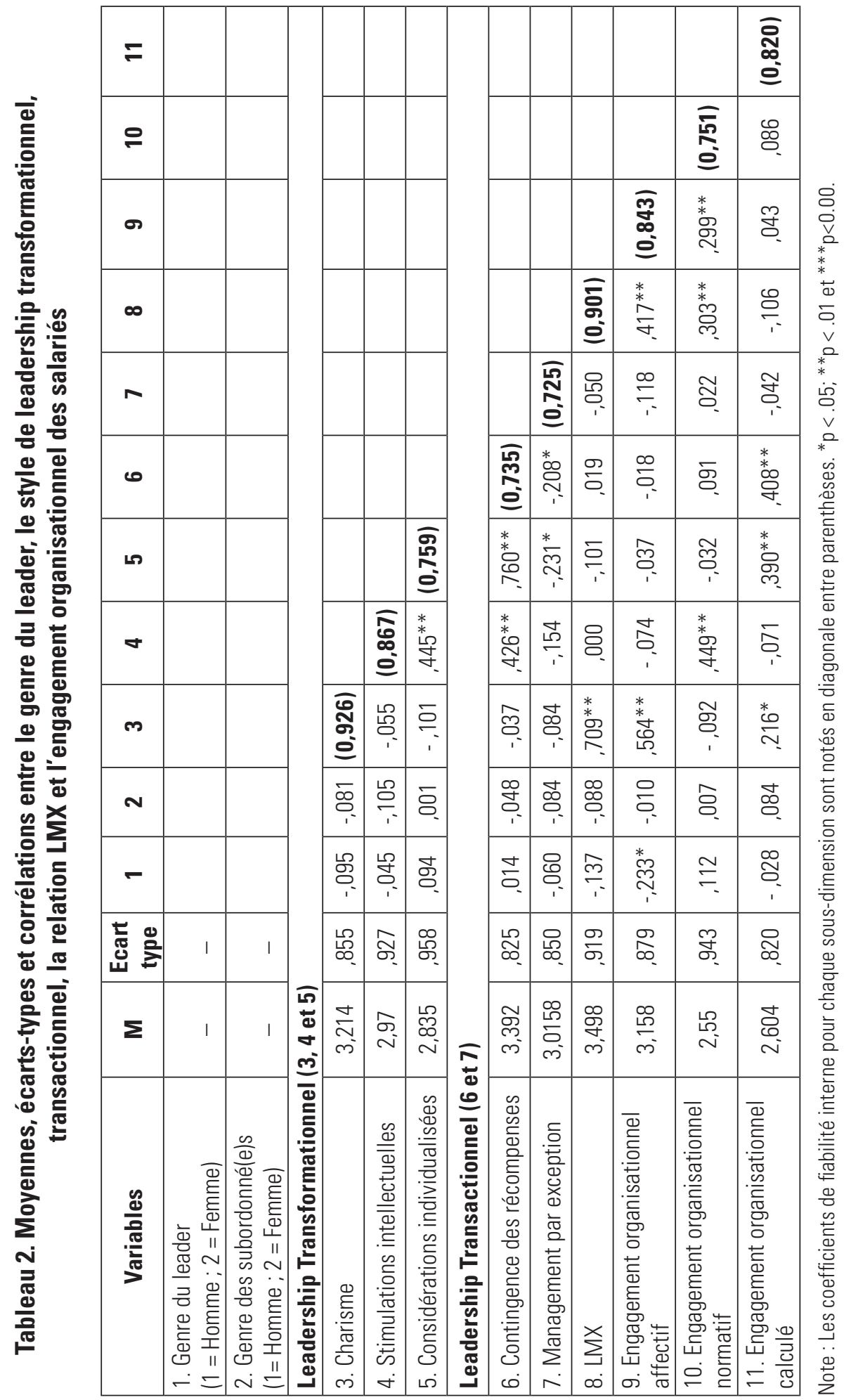




\section{Tableau 3. Régression linéaire hiérarchique modérée des effets du leadership transformationnel, transaction et du LMX en fonction du genre du leader sur l'engagement organisationnel des salariés}

\begin{tabular}{|c|c|c|c|c|c|c|c|c|c|}
\hline \multirow{3}{*}{ Variables indépendantes } & \multicolumn{3}{|c|}{ Engagement affectif } & \multicolumn{6}{|c|}{ Engagement normatif Engagement calculé } \\
\hline & \multicolumn{9}{|c|}{ Model 1 Model 2 Model 3 Model 1Model 2Model 3Model 1Model 2Model 3} \\
\hline & $\beta^{\mathrm{a}}$ & $\beta^{\mathrm{a}}$ & $\beta^{\mathrm{a}}$ & $\beta^{\mathrm{a}}$ & $\beta^{\mathrm{a}}$ & $\beta^{\mathrm{a}}$ & $\beta^{\mathrm{a}}$ & $\beta^{\mathrm{a}}$ & $\beta^{\mathrm{a}}$ \\
\hline \multicolumn{10}{|l|}{ Étape 1 Variables de contrôle } \\
\hline Genre supérieur &,$- 176^{*}$ &,- 152 &,- 146 & ,125 &, 170 & ,158 &, 004 & 065 & ,068 \\
\hline Genre du subordonné &,- 075 &,- 033 &,- 051 & ,096 &, 156 & ,096 &,$- 189 *$ &,- 144 &,- 158 \\
\hline Âge du leader &,- 104 &,- 063 &,- 080 & ,099 & ,096 &, 066 &,- 165 &,- 084 & ,082 \\
\hline Âge du subordonné & $474^{* *}$ &, $358^{* *}$ &, $298 * *$ &,- 004 & 134 &, 113 &, 141 & ,085 &,- 097 \\
\hline Ancienneté du leader &,- 078 &,- 056 & ,050 &,- 010 &,- 052 & ,023 &, 168 & , 198 & ,270 \\
\hline Ancienneté dyade &,- 170 &,- 123 &,- 176 &,- 037 &,- 005 &,- 129 &,- 090 &,- 055 &,- 126 \\
\hline$\Delta R^{2}$ & ,191 & & &, 030 & & &, 075 & & \\
\hline $\mathrm{R}^{2}$ & , 191 & & &, 030 & & & 075 & & \\
\hline Fglobal & $4,092 * * *$ & & &, 527 & & & 1,407 & & \\
\hline \multicolumn{10}{|l|}{ Étape 2 Style de leadership } \\
\hline Charisme & &, $455^{* * *}$ &, $438^{* * *}$ & &, 000 & ,048 & &,- 025 &,- 034 \\
\hline Stimulations intellectuelles & &,- 048 &,- 022 & &, $402^{* * *}$ &, $389^{* * *}$ & &,- 090 &,- 019 \\
\hline Considérations individualisées & &, 138 & 100 & &, 036 & ,022 & &,- 133 &,- 089 \\
\hline Contingences récompenses & &,- 077 &,- 101 & & ,245 & , 198 & & ,233 &, 139 \\
\hline MBE & &,- 079 &,- 096 & & , 108 &, 065 & & ,032 &, 020 \\
\hline LMX & &, 053 &, 064 & &,- 047 &,- 082 & &, $296^{*}$ &, $305^{*}$ \\
\hline$\Delta \mathrm{R}^{2}$ & & $246 * * *$ & & &, $301^{* * *}$ & & &, 102 & \\
\hline $\mathrm{R}^{2}$ & & .437 & & &, 330 & & & 177 & \\
\hline F global & & $6,34^{* * *}$ & & & $4,029 * *$ & & & 1,756 & \\
\hline \multicolumn{10}{|l|}{ Étape 3 Effet modérateur } \\
\hline Charisme $\mathrm{x}$ genre & & &, 112 & & &, 077 & & &,- 102 \\
\hline Stimulations intellectuelles $\mathrm{x} g$ & genre & &, 066 & & & 102 & & & 206 \\
\hline Considérations individualisées & $\mathrm{x}$ genre & & ,077 & & &,$- 285^{*}$ & & & 019 \\
\hline Contingences récompenses x g & genre & &,- 042 & & &, 147 & & &,- 076 \\
\hline MBE x genre & & &,- 146 & & &,$- 221^{* *}$ & & &,$- 277^{* *}$ \\
\hline$\underline{L M X} \times \underline{\text { genre }}$ & & &, 073 & & &, 010 & & &, 022 \\
\hline$\Delta R^{2}$ & & & ,058 & & & ,072 & & &, $119^{*}$ \\
\hline $\mathrm{R}^{2}$ & & & ,495 & & & ,403 & & & ,295 \\
\hline F global & & & $5,009 * * *$ & & & $3,445^{* *}$ & & & $2,143^{* *}$ \\
\hline
\end{tabular}

Note. $\quad{ }^{*} p<.05 ;{ }^{* *} p<.01 ;{ }^{* *} p<.001 ; n=111$. Les coefficients de régression standardisés sont reportés. Les variables du leadership transformationnel, transactionnel et LMX ont été centrées. Le genre a été recodé selon la procédure d'Aiken et West (1991) en - 1 pour les hommes et 1 pour les femmes. 
Aiken et West (1991), nous avons centré ${ }^{6}$ les dimensions du leadership ainsi que les termes de l'interaction entre le genre du leader et le style de leadership, et ce, dans le but de réduire la multi-colinéarité entre l'effet principal et l'interaction (Aguinis, 2004, chapitre 3). Nous avons également recodé les variables catégoriques, liées au genre ${ }^{7}$, pour faciliter l'interprétation des résultats. Afin de répondre aux hypothèses de recherche, trois régressions hiérarchiques ont été réalisées (tableau 3). Le premier modèle introduit les variables de contrôle ; le deuxième intègre les styles de leadership transformationnel, transactionnel et le LMX ; enfin, le troisième inclut le produit du terme multiplicatif entre le genre du leader et les dimensions du style de leadership, ceci afin de tester les hypothèses de recherche 5 et 6 .

\section{Déterminants de l'engagement affectif}

Le tableau 3 présente les résultats des analyses de régression. Le modèle 1 suggère que l'âge du répondant ainsi que le genre du leader influencent significativement son engagement affectif $(\beta=, 474 ; p<.01 ; \beta=-, 176 ; p<.05)$. Toutefois, il est important de noter que la variable genre du leader n'a aucune influence significative sur l'engagement affectif des salariés, dans les modèles de régression suivants. Le modèle 2 , qui est statistiquement significatif $(F(12,98)=6,342 ; p<.001)$, suggère que le charisme $d u$ leader ( $\beta=, 455 ; p<.001)$ influence significativement l'engagement affectif des salariés. Le test de l'effet modérateur du genre entre le style de leadership et l'engagement affectif n'est pas concluant, puisque les résultats statistiques ne sont pas significatifs $\left(\Delta R^{2}=0,058 ; p=n . s\right)$. II apparaît donc que l'hypothèse 5a n'est pas validée, puisque le genre du leader ne semble pas avoir d'effet modérateur entre le style de leadership et l'engagement affectif des salariés.

Toutefois, les résultats révèlent que, quel que soit le sexe du leader, " le charisme " de celui-ci influence positivement l'engagement affectif des salariés. Les données révèlent également que l'âge du répondant (i.e., le subordonné) semble avoir une influence significative sur son engagement affectif envers son entreprise. En effet, les subordonnés plus âgés tendent à être davantage engagés affectivement envers leur organisation. Ces résultats rejoignent les conclusions de Mathieu et Zajac (1990), lesquelles indiquent que l'âge et l'engagement affectif sont liés. II semble donc que plus les subordonnés perçoivent leur leader comme étant un modèle à suivre, plus leur engagement affectif envers leur entreprise est intense. Cependant, le genre du leader ne démontre aucun effet modérateur des liens entre le leadership et l'implication affective des salariés.

[6] Les scores centrés ont été obtenus par la soustraction de la moyenne respective de chacune des dimensions (Aiken et West, 1991).

[7] Les variables catégoriques liées au genre ont été recodées : les hommes sont codés - 1 et les femmes sont codées 1. 


\section{Déterminants de l'engagement normatif}

Le modèle 1 indique qu'aucune des variables de contrôle introduites dans le modèle de régression n'influence significativement l'implication normative des salariés. II apparaît, dans le deuxième modèle, que l'introduction des variables liées aux styles de leadership impacte significativement la variance de l'engagement normatif. En effet, Le modèle 2 est statistiquement significatif $(F(12,98)=4,029 ; p<.001)$. Plus particulièrement, la dimension "stimulations intellectuelles " détermine significativement l'engagement normatif des salariés $(\beta=, 402 ; p<.001)$. Cela induit que, plus les leaders incitent leurs subordonnés à l'émulsion intellectuelle, plus leurs collaborateurs déploient un engagement de type normatif vis-à-vis de leur organisation. Le modèle 3, qui teste l'effet modérateur du genre, souligne que les interactions " stimulations intellectuelles " et genre du leader $(\beta=, 402 ; p<.01)$ puis management par exception et genre du leader $(\beta=, 402 ; p<.01)$ influencent significativement l'engagement normatif des salariés. Cependant, ces résultats ne peuvent donner lieu à des conclusions puisque $\Delta R^{2} n^{\prime}$ est pas significative $\left(\Delta \mathrm{R}^{2}=0,072 ; p=n s\right)$. Ainsi, l'ajout des termes d'interaction entre le genre et le style de leadership n'apporte pas un effet statistiquement significatif pour expliquer la variance de l'engagement normatif des salariés. L'hypothèse $5 b$ n'est donc pas validée.

\section{Déterminants de l'engagement calculé}

Les résultats du premier modèle de régression suggèrent que le genre du répondant influence l'engagement calculé des salariés $(\beta=-189 ; p<.05)$. Cependant, nous ne pouvons conclure puisque le modèle global ne présente pas de résultats statistiquement significatifs $(F(6,104)=1,407 ; p=n . s)$. Le deuxième modèle signale que le LMX influence positivement l'engagement calculé des salariés ( $\beta=296 ; p<.05)$. Néanmoins, ce résultat, surprenant, doit être interprété avec précaution, puisque le modèle global n'est pas statistiquement significatif ( $F(12,98)=1,756 ; p=n$.s). Quant au troisième modèle, les résultats démontrent qu'il est statistiquement significatif $(F(18,92)=2,143$; $p<.01)$. De plus, l'apport du terme d'interaction entre le genre du leader et le style de leadership est statistiquement significatif $\left(\Delta R^{2}=, 119 ; p<.05\right)$, et révèle que l'ajout de cette interaction explique $11,9 \%$ de plus, à la variance de l'engagement calculé des salariés. Plus particulièrement, l'interaction entre le genre du leader et le « management par exception " $(\beta=-, 277 ; p<.01)$ est statistiquement significative pour expliquer I'implication calculée des salariés. Ainsi, le genre du leader modère la relation entre le $M B E^{8}$ et l'engagement calculé des salariés. L'hypothèse 6 est donc validée.

Afin de mieux interpréter ces résultats nous avons réalisé un graphique, suivant les recommandations de Cohen et Cohen (2003). La figure 1 montre que, lorsque les femmes

[8] Le terme MBE est une abréviation de la dimension « management par exception ». 
leaders déploient un style de leadership de type MBE de manière élevée, c'est-à-dire en se désengageant fortement de leur fonction de leader, leurs collaborateurs se désinvestissent de façon calculée vis-à-vis de leur organisation. Cependant, lorsqu'un homme leader déploie les mêmes comportements, ses subordonnés maintiennent un niveau constant d'engagement calculé envers leur entreprise.

\section{Figure 1. Le genre du leader comme modérateur de la relation entre le MBE et l'engagement calculé des salariés}

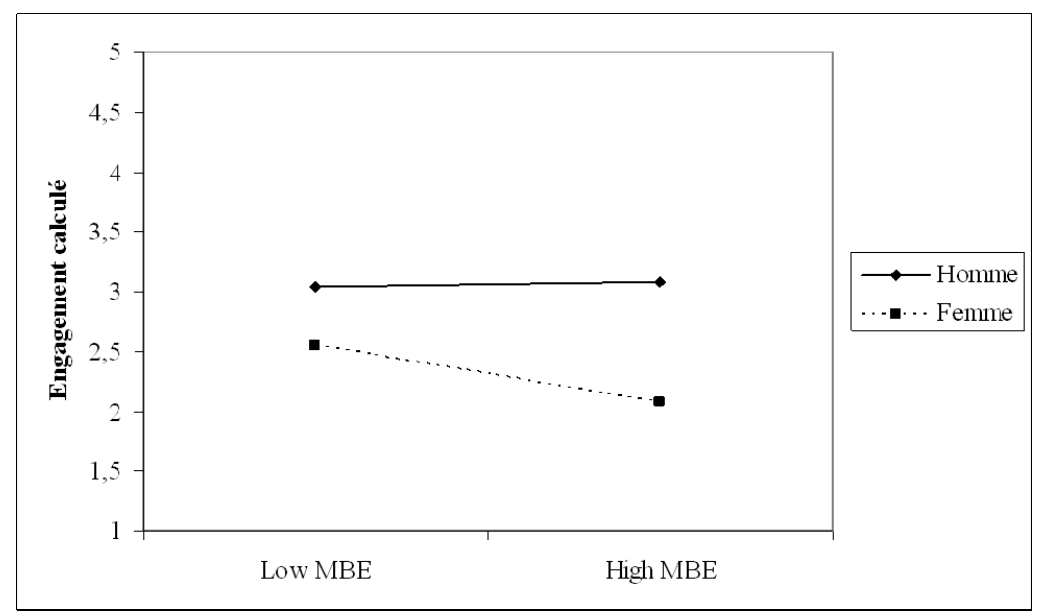

\section{Discussion}

L'objectif de cette communication est d'examiner les liens éventuels entre genre, style de leadership et engagement organisationnel des salariés. Tandis que la méta-analyse a mis au jour l'impact significatif du genre sur le style de leadership transformationnel et sur la dimension " contingence des récompenses " du leadership transactionnel (Eagly et al., 2003), nos résultats n'ont, quant à eux, relevé aucune incidence significative entre ces deux concepts.

En revanche, selon nos données, le style de leadership, et plus particulièrement le management par exception, suscite auprès des subordonnés un engagement calculé différencié en termes d'intensité, selon le genre du leader. Lorsque les managers hommes déploient ce type de leadership, leurs collaborateurs génèrent tout de même une implication calculée vis-à-vis de leur organisation. Tandis que lorsqu'une femme dirigeante adopte ce comportement de désengagement, ses collaborateurs se désinvestissent eux aussi de leur organisation (figure 1). Nous supposons que cette conduite est associée au rôle masculin, traditionnellement considéré comme directif. Ainsi, il semblerait normal pour un homme leader de n'intervenir, en tant que responsable, que quand les problèmes deviennent extrêmement importants. Néanmoins, une femme ne peut pas se 
permettre un leadership attentiste, auquel cas, ses subordonnés se désengageraient fortement de leur organisation.

Nos résultats mettent en exergue le fait que les hommes manifestant ce type de leadership suscitent un engagement positif de la part des salariés, tandis que les femmes qui l'utilisent, génèrent un désengagement auprès de leurs subordonnés. Selon la théorie du rôle social (Eagly, 1987) et les théories implicites du leadership (Phillips et Lord, 1981), les salariés attendent de leur supérieur des comportements particuliers, conformes à leur genre. Les femmes sont notamment encouragées à adopter des styles de management participatifs, de collaboration avec leurs subordonnés (Eagly et al., 1990), tandis que les hommes leaders sont stéréotypés comme étant plus directifs (Eagly et al., 1990). Les résultats soulignent ce que les chercheurs nomment une « incongruité du rôle de genre ", dans la mesure où les femmes, en adoptant le style de leadership " management par exception », transgressent les attentes habituellement associées à leur rôle, ce qui génère, auprès de leurs subordonnés, des attitudes négatives (Eagly, 1987).

Ainsi, une femme leader, en déployant des conduites de management passif, suscite de la part de ses subordonnés un désengagement beaucoup plus fort que lorsque c'est un homme leader qui adopte ces mêmes conduites. Les résultats tendent à souligner une sexualisation des attentes des subordonnés vis-à-vis de leurs dirigeants. Ils espèrent trouver, chez les femmes managers, des comportements de leadership transformationnel. Lorsqu'elles ne se conforment pas à ces attentes, leurs subordonnés se désengagent de l'organisation. En revanche, lorsqu'un homme agit ainsi, ses subordonnés restent tout de même impliqués dans l'organisation.

En dépit de ces différences liées au genre, les résultats soulignent une similarité des comportements des subordonnés en fonction du genre du leader. Les dimensions affective et normative de l'engagement des salariés sont déterminées par le " charisme " et " les stimulations intellectuelles » et ce, quel que soit le genre du manager. Nos résultats remettent donc en cause l'idée d'un " avantage féminin " au leadership, puisque, quel que soit le sexe du leader, les attitudes du leader, perçues comme exemplaires et stimulantes intellectuellement, influencent positivement l'engagement affectif et normatif des salariés.

Malgré l'absence de différence, au regard du genre du leader sur le style de leadership, nous constatons néanmoins qu'il influe sur le type d'engagement des salariés. Ainsi, il apparaît que, si le type de management ne dépend pas du genre, celui-ci a toutefois une incidence différente sur les attentes ainsi que sur les perceptions des salariés.

\section{LIMITES ET IMPLICATIONS MANAGÉRIALES}

Les résultats de notre étude comportent deux principales limites. D'une part, la taille réduite de l'échantillon $(N=111)$ ne permet pas d'atteindre la représentativité requise. 
D'autre part, celui-ci gagnerait à être davantage diversifié, tant en termes de secteur d'activité que de position hiérarchique (cadre intermédiaire, top manager, cadre dirigeant) ; ce qui consoliderait l'observation de l'impact des facteurs organisationnels sur le style de leadership (Gardiner et Tiggerman, 1999).

Néanmoins, ces résultats nous autorisent d'appréhender le phénomène du plafond de verre sous l'angle de l'incompréhension. En effet, en dépit d'une absence de compétences managériales spécifiques, déterminées par le genre, les femmes demeurent pourtant sous- représentées aux positions de top management. En définitive, notre analyse infirme l'hypothèse d'une incompétence féminine à diriger des équipes, puisque le genre ne constitue guère une variable significative du style de leadership. Cependant, les perceptions stéréotypées perdurent ; le genre du leader demeure, en effet, une variable déterminante du type d'engagement organisationnel du salarié. Ces conclusions représentent pour les entreprises un levier d'action. Au regard de celles-ci, le genre du manager constitue une variable non déterminante des compétences au leadership. II ressort clairement des résultats de nos travaux que les supérieurs hiérarchiques ont tout intérêt à développer des comportements de leadership transformationnel et tout particulièrement de charisme et de stimulations intellectuelles lesquels génèrent une incidence positive sur l'engagement affectif et normatif des salariés. Notre étude confirme les précédentes menées sur cette thématique (Bono et Judge, 2003 ; Dumdum et al., 2002). De plus, elle nous indique que les conduites, inspirées par le leadership « management par exception ", suscitent des outputs organisationnels différenciés selon le sexe du leader. Lorsqu'une femme échoue dans ses fonctions de leadership, ses collaborateurs se désengagent de manière calculée vis-à-vis de leur entreprise. Alors que, lorsqu'un homme échoue dans ses fonctions de leadership, ses collaborateurs s'impliquent, tout de même, dans l'entreprise, de façon calculée. Ces résultats semblent signifier que l'échec de la part d'une femme, en tant que leader, génère un désengagement plus intense de ses collaborateurs que l'échec de la part, d'un homme leader. II paraît donc nécessaire, pour les entreprises, de former leurs responsables au leadership afin qu'ils puissent adopter des comportements de "stimulations intellectuelles " et être perçus comme charismatiques, qui, quel que soit le sexe du leader, génèrent un engagement positif envers l'organisation. II s'avère également important de rompre avec les stéréotypes liés au genre du manager, qui, semble-t-il, perdurent encore aujourd'hui.

\section{Remerciements}

Nous tenons à remercier l'ensemble des évaluateurs anonymes, pour leurs précieux conseils ainsi que pour leurs relectures avisées. 


\section{RÉFÉRENCES}

ACKER, J. (1990). "Hierarchies, jobs, bodies: a theory of gendered organizations", Gender and Society, Vol. 4.

AGUINIS, H. (2004). Regression analysis for categorical moderators. New York: Guilford.

AGUINIS, H. \& GOTTFREDSON, R. K. (2010). "Best-practice recommandations for estimating interraction effects using moderated multiple regression", Journal of Organizational Behavior, 31, 776-786.

AIKEN, L. S. \& WEST, S. G. (1991). Multiple Regression: Testing and interpreting interactions, Newbury Park, CA: Sage.

ALLEN, N. J. \& MEYER, J. P. (1990). "The measurement and antecedents of affective, continuance and normative commitment to the organisation", Journal of Occupational Psychology, 63, 1-18.

ALLEN, N. J. \& MEYER, J. P. (1996). "Affective, continuance and normative commitment to the organization: an examination of construct validité", Journal of Vocational Behavior, 49, 252-276.

ARNOLD, K. A., BARLING, K., \& KELLOWAY, E. K. (2001). “Transformational leadership or the iron cage: which predicts trust, commitment and team efficace?", Leadership and organization development journal, 22, 315-320.

AVOLIO, B. J., BASS, B. M., \& JUNG, D. (1999). "Re-examining the components of transformational and transactional leadership using the Multifactor Leadership Questionnaire", Journal of Occupational and Organizational Psychology, 72, 441-462.

BASS, B. M. (1985). Leadership and performance beyond expectations, New York: Free Press.

BASS, B. M., \& AVOLIO, B. J. (1997). “Full range leadership development: Manual for the Multifactor Leadership Questionnaire", California: MindGarden Inc.

BLANCHARD, S. LEFLEUVE, N., \& MESTO, M. (2009). « Les femmes cadres et dirigeantes d'entreprise en Europe ", VIm 151, Genre et Pouvoir en Europe.

BONO, J. \& JUDGE, T. (2003). "Self concordance at work: toward understanding the motivational effects of transformational leadership", Academy of Management Journal, 46, 554-571.

BURNS (1978), Leadership, New York: Harper \& Row.

CARLESS, S. A. (1998). "Gender Differences in Transformational leadership: An examination of superior, leader and subordinate perspective", Sex Roles, 39.

COHEN, J. \& COHEN, P. (2003). Applied multiple regression/correlation analysis for the behavioral sciences, Hillsdale, NJ: Erlbaum. 
CORNET, A. \& BONNIVERT, S. (2008) « Leadership et Genre ", dans Cornet, A., Lauffer, J., et Belghiti-Mahut, S., GRH et Genre : les défis de l'égalité homme-femme, Paris, Vuibert, p. 125-137.

DAVIDSON, M. \& BURKE, R. (2000). Women in Management: Current Research Issues, VIm II, Thousands Oaks, CA, Sage Publication.

DRUSKAT, V. U. (1994). "Gender and Leadership style: Transformational and transactional leadership in Roman Catholic Church", Leadership Quarterly, Vol. 5, 99-119.

DUMDUM, U. R., LOEWE, K. B., \& AVOLIO, B. (2002), "A meta analysis of transformational and transactional leadership correlates of effectiveness and satisfaction: an update and extension", in B. J. AVOLIO \& F. J. YAMMARINO (Eds), Transformational and charismatic leadership: The road ahead, Oxford, U.K.Elsevier Science.

EAGLY, A. H. (2007). "Female leadership advantage \& disadvantage: Resolving the contradictions", Psychology of Women Quarterly, vol. 31, p. 1-12.

EAGLY, A. H. (1987), Sex differences in social behavior: A social-role interpretation, Hillsdale, NJ: Erlbaum.

EAGLY, A. H., JOHANNESEN-SCHMIDT, M.C., \& VAN ENGEN, M.L. (2003). "Transformational, Transactional, and Laissez Faire Leadership Styles: A Meta Analysis Comparing Women and Men", Psychological Bulletin, Vol. 29, No. 4, 569-591.

EAGLY, A. H. \& JOHNSON, B. T. (1990). "Gender and Leadership Style: A meta analysis", Psychological Bulletin, 108, 233-256.

FELDMAN, D. C. (1976). "A contingency theory of socialization", Administrative Science Quarterly, 21, 433-452.

GARDINER, M. \& TIGGEMANN, M., (1999). "Gender differences in leadership style, job stress and mental health in male and female dominates industry", 72, Journal of Occupational and Organizational Psychology, 72.

GERSTNER, C. R. \& DAY, D. V. (1997). "Meta analytic review of leader-membre exchange theory: correlates and construct issues", Journal of Applied Psychology, 82, 827-844.

GRAEN, G. B. \& UHL-BIEN, M. (1991). "The transformation of professionals into selfmanaging and partially self-designing contributions: Toward a theory of leadership making", Journal of Management Systems, 3(3), 33-48.

GRAEN, G. B. \& UHL-BIEN, M. (1995) "Development of leader-member exchange (LMX) theory of leadership over 25 years: Applying a multi-level, multi-domain perspective", Leadership Quarterly, Vol. 6, 219-247

GRAEN, G. B. \& CASHMAN, J.(1975). "Role making model of leadership in formal organizations: A developmental approach", in J. G. Hunt \& L. L. Larson (Eds), Leadership frontiers (pp. 143). Kent, OH: Kent State University Press.

HAYWARD, 0., GOSS, M., \& TOLMAY, R., (2004). The relationship between transformational and transactional leadership and employee commitment. Grahamstown, SA: Rhodes University. 
HELGESEN, S. (1990). The Female Advantage: Women's Way of Leadership, Doubleday, New York, NY.

KANTER (1977). Men and women of the corporation. New York: Basic Books.

KENT, A. \& CHELLADURAI, P. (2001). "Perceived transformational leadership, organizational commitment, and citizenship behavior: a case study in intercollegiate athletics". Journal of sport management, 15 (2), 135-159.

KOLB, J. A. (1999). "The effect of Gender role attitude toward leadership, and self-confidence on leader emergence: Implications for leadership development", Human Resource Development Quarterly, Vol. 10, No. 4, p. 305-320.

KOMIVES, S. R. (1991). "The relationship of same - and cross - gender work pairs to staff performance and supervisor leadership in residence hall units", Sex Roles, Vol. 24, p. 355-363.

KOUZES, J \& POSNER, B. (1990) "Leadership Practices Inventory (LPI): A self assessment and analysis", Peiffer \& Co., San Diego, C.A.

KUNHNERT, K.W. \& LEWIS, P. (1987). "Transactional and Transformational leadership: A constructive/developmental analysis", Academy of Management Review, Vol. 12, No. 4, p. 648-657.

MACCOBY \& JACKLIN (1974), The psychology of sex differences, Stanford, CA: Stanford University Press.

MAHER, K. J. (1997). “Gender related stereotypes of transformational and transactional leadership", Sex Roles, Vol. 3, p. 209-225.

MANDELL, B. \& PHERWANI, S. (2003). "Relationship between Emotional intelligence and Transformational leadership style: A gender comparison", Journal of Business and Psychology, Vol. 17, No. 3, p. 387-404.

MATHIEU, J. E. \& ZAJAC, D. M. (1990), "A review and meta analysis of the antecedents, correlates and consequences of organizational commitment", Psychological Bulletin, 108, 171-194.

MEYER, J. P., ALLEN, N. J., \& C. A. SMITH. (1993). “Commitment to organizationsand occupations: Extensions and test of a three-component conception", Journal of Applied Psychology, 78, 538-551.

MOWDAY, R. T., PORTER, L. W., \& STEERS, R. M. (1982). Employee-organization linkages. New York: Academic Press.

NEVEU, V. (2004), " La confiance organisationnelle : une approche en terme de contrat psychologique ", Thèse de Doctorat en Sciences de Gestion, Université Paris I Panthéon-Sorbonne.

NIEVA, V. F. \& GUTEK, B. A. (1981). "Sex effects on evaluation", Academy of Management Review, VIm 5, 267-276.

NORTHOUSE, P (2010). Leadership: Theory and Practices, New York: Sage Publications. 
NUNNALlY, J. (1967), Psychometric Theory, New York: McGraw-Hill.

PHILLIPS, J. S. \& LORD, R. G. (1981). "Causal attributions and perceptions of leadership", Organizational behavior and Human Performance, 28, 58-83.

POUNDER, J. S. \& COLEMAN, M. (2002) "Women - better leader than men? In general and educational management it still 'all depends'", Leadership \& Organizational Development Journal, 23, 3/4, p. 122.

ROSENER, J. B. (1990). "Ways women lead", Harvard Business Review, Vol. 68, p. 119-125.

SAINT-MICHEL, S. (2010). "L'impact du genre sur le style de leadership : I'importance d'introduire les traits de personnalité des leaders ", Revue Internationale de Psychosociologie, 40, 181-203.

SCANDURA, T. A. \& GRAEN, G. B. (1986). "When managers decide not to decide autocratically: An investigation of leader-member exchange and decision influence", Journal of Applied Psychology, Vol. 71, p. 579-584.

SHAMIR, B., ZAKAY, E., BREININ, E., \& POPPER, M. (1998). “Correlates of charismatic leader behavior in military units: subordinates'attitudes, unit characteristics and superior's appraisal of leader performance", Academy of Management Journal, 41, 387-409.

TERBORG, J. R. (1977). "Women in management: A research review", Journal of Applied Psychology, 1977, 62, 647-664.

TETT, R. P. \& MEYER, J. P. (1993). "Job satisfaction, organizational commitment, turnover intention and turnover: path analyses based on meta analytic findings", Personnel Pyschology, Vol. 46, p. 259.

VAN ENGEN, M. L., VAN DER LEEDEN, R., \& WILLEMSEN, T. M. (2001). "Gender, context and leadership styles: A field study", Journal of Occupational \& Organisational Psychology, Vol. 74, p. 581-598.

VARMA, A. \& STROH, L. K. (2001). "The impact of same sex LMX dyads on performance evaluations", Human Resource Management, Vol. 40, No. 4, p. 309-320.

VECCHIO, R. P. (2002). "Leadership and gender advantage", Leadership Quarterly, Vol. 13, p. 643-671.

VECCHIO, R. P. \& BRAZIL, D. M. (2007). "Leadership and Sex-similarity: A comparaison in military setting", Personel Psychology, 60, 303-335.

WAJCMAN (1998), Managing like a man: Women and men in corporate management, University Park, PA: Pennyslvania State University Press.

WANOUS, J. E. (1977). "Organizational entry: Newcomers moving from outside to inside", Psychological Bulletin, 84, 601-618.

YAMMARINO, F. J., DUBINSKY, A. J., COMMER, B. L., \& JOLSON, M. A. (1997). “Women and Transformational and Contingent Reward leadership: A multiple levels of analysis perspective", Academy of Management Journal, Vol. 40, No. 1, p. 205-222. 